\title{
Formation, Crystal Growth and Colour Appearance of Mimetic Tianmu Glaze
}

\author{
Chang-Yang Chiang ${ }^{\mathrm{a}}$, Heather F. Greer ${ }^{\mathrm{a}}$, Ru-Shi Liu ${ }^{\mathrm{b}}$, Wuzong Zhou ${ }^{\mathrm{a}, *}$ \\ ${ }^{a}$ School of Chemistry, University of St Andrews, St Andrews, Fife KY16 9ST, UK. \\ ${ }^{b}$ Department of Chemistry, National Taiwan University, Taipei 106, Taiwan
}

\author{
* Corresponding author. \\ Telephone: +44 1334 467276, Fax: +44 1334 463808, E-mail address: wzhou@st- \\ andrews.ac.uk (W.Z. Zhou)
}

\begin{abstract}
:
Mimetic Tianmu glaze has been synthesized and analysed by using X-ray

diffraction, energy-dispersive X-ray spectroscopy, scanning electron microscopy and transmission electron microscopy. It was found that the main body of the glaze was amorphous aluminium silicate with many embedded polycrystalline spherical particles of metal oxides containing manganese, cobalt, vanadium, bismuth and tungsten. Two dimensional spinel dendrites crystals of manganese, cobalt and aluminium oxide formed on the surface of the glaze. The formation mechanism of the microstructures in the Tianmu glaze is proposed. The colour appearance of the glaze has also been discussed. It has been found that the crystal thickness dependant light interference could be an important factor for the appearance of rainbow-like colour in the glaze layer.
\end{abstract}


Keywords: A: Sintering, B: electron microscopy, D: Glass, D: Spinels.

\section{Introduction}

Tianmu bowls (also named Tenmoku or Temmoku bowls) are a variety of tea bowls originally made in China 800 years ago, as one of the most exquisite ancient Chinese chinawares produced in Jian Kilns [1]. They were coated with a layer of glaze (henceforth called Tiamnu glaze), displaying a black, brown or grey background and fantastic patterns after firing in a kiln. Based on the characteristics of their colour appearance and fine patterns, the bowls were classified into different species such as Oil Spot Tianmu, Hare's Fur Tianmu, Yao Bian (Yohen) Tianmu or Leaf Tianmu, etc. These bowls were popular and reached their peak production during the Southern Song Dynasty (ca. 1127-1279). Most of the retained finest works, including a few listed in the National Treasure of Japan [2], were made in that period. Among the four traditional evaluation criteria for Chinese tea, colour, smell, taste and shape, the Tianmu bowls are believed to affect three of them. In the dark background of Tianmu bowls, the white tea leafs, which was popular in Song Dynasty, give a high contrast, wonderful colour appearance and a clear shape. It is also believed by some people that the taste of tea using Tianmu bowls is purer and milder [3].

However, with the passage of time, production of the Tianmu bowls did not move 
forward and the details of the manufacturing process have been lost. It was rare that research was carried out during that period and it was not until recent decades that the beauty of Tianmu bowls attracted peoples' attention again. It is obvious that the most important part of the Tianmu bowls is their glaze. To reproduce the appearance, the components of ancient Tianmu bowls have been characterised mainly by diffraction and microscopy based techniques, in addition to the studies of the relevant ancient literature. By trial and error, some Tianmu bowls have been imitated with similar characteristics to the ancient products. More tea bowls with beautiful appearance of textural patterns and amazing colours have also been produced, and are marketed nowadays as Tianmu bowls (Fig. 1). The patterns and colours are quite similar to the ancient bowls $[4,5]$.

Based on the analysis of ancient Tianmu bowls, it is known that the Tianmu glaze is one kind of crystalline glaze with a glass-like body and textured crystals on the surface. These textures, except several special types which can be manipulated using a template, e.g. the leaf patterns on Leaf Tianmu) [6], form randomly. Therefore, each bowl has a unique pattern. The formation of these self-formed textures, for example, the round spots on Oil Spot Tianmu or the straight lines on Hare's Fur Tianmu, have previously been studied and compared to each other [7-9]. It was found that the formation of different textures is related to liquid phase separation during the firing 
process. The separated phases could aggregate and form in different shapes such as round spots or lines, depending on multi-effects from the glaze system and the environment in kilns, followed by crystallization forming the final textures. At the same time, the colour appearance on the bowls can result from different types of crystals. In some cases, on the other hand, the morphology of the textures itself might be the dominant factor for the colour appearance, as several researchers proposed that the rainbow-like colour on Yao Bian Tianmu might be caused by a thin layer on the glaze surface in which the light interference happens. Further experimental research showed that the formation of the crystalline textures could be influenced by many factors such as the viscosity of the glaze, impurities in the kiln, firing process, thickness of the glaze layer and curvature of the bowls [10-12].

Although the appearance of Tianmu bowls is quite different from each other, the crystal structures forming in the glaze are relatively simple. Until now, iron oxides are the most common crystalline components found in different species of Tianmu bowls, either ancient or imitation products. Many researchers have achieved products with appearance similar to the ancient bowls based on the use of iron. Nowadays, on the other hand, many potters also use manganese- and cobalt-containing raw materials to make Tianmu-type glazes, or even richer in colour. Using these compositions of glaze, 
with further modification of the manufacturing process, is now a common way to

achieve the rainbow-like colours similar to Yao Bian Tianmu. Although many Mn- and Co-containing products have already been made, the formation of these glazes, including the crystallization process, the phase separation, the microstructures, and the colour appearance, is not yet investigated.

Herein, a Tianmu glaze is prepared by adding manganese and cobalt sources and its microstructures are studied. The glaze components and crystal morphology are analyzed by using optical microscopy, scanning electron microscopy (SEM) and transmission electron microscopy (TEM), while the crystalline phases are determined by powder XRD and high resolution TEM (HRTEM). The formation mechanism of the two-dimensional surface crystals and colour appearance are discussed.

\section{Experimental Section}

\section{Glaze preparation}

In the present work, potassium feldspar (Tyng Hann Industrial Co. Ltd), silicon oxide (Sibelco Asia Pte Ltd), manganese oxide, ammonium metavanadate (Yir Ming Kiln Co. Ltd), cobalt oxide (Umicore), bismuth oxide, tungsten oxide and magnesium carbonate (Nihon Shiyaku Industries, Ltd), and ISOBOM (Kuraray) (one kind of binder 
made by isobutylene and maleic anhydride) were used to make the glaze. The formula, components, amount and possible function of each raw material are shown in Table 1, according to a recipe given by Mr. Yi-Zhi Qiu of the Shang Liu Pottery Club.

After weighing the raw materials, they were added to a ball mill with alumina milling balls and distilled water. After milling for $15 \mathrm{~min}$, the glaze was sprayed on a porcelain substrate using a spray bottle and the porcelain was then transferred into an electric kiln. The firing program was as follows, the temperature was raised at $4{ }^{\circ} \mathrm{C} / \mathrm{min}$ to $800{ }^{\circ} \mathrm{C}$, followed by $2{ }^{\circ} \mathrm{C} / \mathrm{min}$ to $1160^{\circ} \mathrm{C}$, and finally $1{ }^{\circ} \mathrm{C} / \mathrm{min}$ to $1260^{\circ} \mathrm{C}$, and was held at $1260{ }^{\circ} \mathrm{C}$ for $20 \mathrm{~min}$. The temperature was then decreased by $2{ }^{\circ} \mathrm{C} / \mathrm{min}$ to 1160 ${ }^{\circ} \mathrm{C}$ and held at $1160{ }^{\circ} \mathrm{C}$ for $2.5 \mathrm{~h}$ before cooling to room temperature at $5{ }^{\circ} \mathrm{C} / \mathrm{min}$. After firing, the samples were cut into suitable sizes for characterization.

\section{Characterization}

Powder XRD was performed on a PANalytical Empyrean diffractometer, using $\mathrm{Cu}$ $\mathrm{K} \alpha$ radiation. Analysis of the XRD patterns was carried out using Highscore plus software. SEM images of the specimens were obtained using either a JEOL JSM-6700F field-emission gun microscope, operating at 1 to $5 \mathrm{kV}$ with gentle mode or a JEOL JSM-5600 SEM with a tungsten filament electron gun. To overcome beam charging 
problem, the specimen surface was coated with a thin gold film using a Quorum Technologies Q150R ES sputter coater/carbon coater prior to SEM analysis. The JEOL JSM-5600 SEM is equipped with an Oxford INCA system for energy dispersive X-ray spectroscopy (EDX), which was applied for examination of the local chemical composition of the specimens. HRTEM images and selected area electron diffraction (SAED) patterns were attained using either a JEOL JEM-2011 electron microscope fitted with $\mathrm{LaB}_{6}$ filament operating at an accelerating voltage of $200 \mathrm{kV}$, a JEOL ARM-200F, JEM-2800 or a FEI Titan Themis S/TEM electron microscope. The microscopes are also equipped with an Oxford EDX system. The TEM and HRTEM images were recorded using a Gatan 794 CCD camera. TEM samples for profile and top views were prepared by FIB on a dualbeam JEOL JIB-4501 and ion-milling on Gatan Model 691 Precision ion polishing system.

\section{Results and discussion}

After firing in a kiln, the glaze displayed a dark-brown base colour with many radial textures on the surface. These textures show a changeable colour from the centre to the edge, which is similar to the colour appearance of Yao Bian Tianmu. The SEM image in Fig. 2(a) shows that each radial texture has a central point from where a 
hexagonal dendrite grows. Fig. 2(b) is an enlarged image showing more details of the branches of the crystal. The centre of the hexagonal pattern is the nucleation site, from which crystal grows out two-dimensionally six principal branches with $60^{\circ}$ uniform inter-branch angles. These dendrites form a plate-like structure on the surface which was observed from a profile view as shown in the first image of Fig. 2(c). The thickness of the dendrite layer is about $90 \mathrm{~nm}$. The measurement of other dimensions of a typical principal branch shows a width of about $1 \mu \mathrm{m}$ and a length of about $3 \mathrm{~mm}$. These very long and thin straight branches imply a highly selective crystal growth direction. Inside the glaze body there are a lot of drop-like spherical clusters.

To understand the elemental distribution in the glaze, EDX elemental mapping was carried out and the results are shown in Fig. 2(c) and Fig. S1 of Supporting Information. The results show that the dendrites on the glaze surface are mainly composed of manganese, cobalt, aluminium (relatively low concentration) and oxygen. This crystal layer is sandwiched between two very thin potassium-rich layers, while the main glaze body is largely formed of silicon, aluminium, potassium and oxygen. The spheres inside the glaze are rich in bismuth and the transition metals, i.e. manganese, cobalt, vanadium and tungsten.

Since the dendrites are the main component in the surface area of the glaze, 
realizing the structure of these dendrites could not only help to understand the growth process, but also the colour appearance of the glaze. One original sample and another with the dendrite layer removed by polishing were analyzed by XRD. The XRD patterns in Fig. 3(a) indicate that the diffraction peaks only come from the original sample, which confirms that the dendrites are the principal crystalline phase, while the main body of the glaze underneath the dendrite layer is basically amorphous. Moreover, all of these peaks, with the coordinated d-spacings of approximately $4.83,2.42,1.61$ and 1.21 $\AA$, show a multiple integer relationship and could be indexed to the (111), (222), (333) and (444) planes respectively of a pseudo-cubic unit cell with the unit cell dimension of ca. $8.37 \AA$, that has been found to be a spinel-type structure as discussed below. This means that the whole crystalline layer are highly orientated with the $(111)_{\mathrm{C}}$ plane parallel to the glaze surface (The subscript C stands for pseudo-cubic unit cell). In order to find out other crystallographic planes from the dendrites, a thin sample with a dendrite crystal lying on the glaze was prepared by ion-milling. Fig. 3(b) is a TEM image showing the morphology of this dendrite with a top view and its corresponding SAED pattern shown in the inset. The diffraction pattern confirms the single crystal nature of the whole dendrite plate. The diffraction spots marked by yellow circles with d-spacings around $2.8 \AA$ can be indexed to the $\{2 \overline{2} 0\}$ planes of a pseudo- 
cubic spinel structure rich in manganese with unit cell parameters close to $8.37 \AA$ [13].

The viewing direction of the SAED pattern is also along the [111]C zone axis, in an agreement with the XRD result. All other TEM results recorded from the dendrites with different viewing directions can also be indexed to this structure. From Fig. 3(b), it is also determined that the highly selected crystal growth directions are six equivalent $<2 \overline{2} 0>$ zone axes, which are perpendicular to the (111) surface.

However, a careful measurement of the SAED pattern reveals that the angles between two adjacent $<2 \overline{2} 0>$ directions are $58^{\circ}$ and $62^{\circ}$, slightly deviated from the ideal hexagonal symmetry. Consequently, the real structure is tetragonal rather than cubic.

Spinel $\left(\mathrm{AB}_{2} \mathrm{O}_{4}\right)$ is a common structure with plenty of isomorphs such as $\mathrm{MgAl}_{2} \mathrm{O}_{4}$ (spinel), $\mathrm{Fe}_{3} \mathrm{O}_{4}$ (magnetite), $\mathrm{Mn}_{3} \mathrm{O}_{4}$ (hausmannite), $\mathrm{CuFe}_{2} \mathrm{O}_{4}$ or $\mathrm{MnCo}_{2} \mathrm{O}_{4}$, etc. They form in nature or can be easily synthesized. The oxygen anions in a spinel form a cubic close-packed structure, in which $1 / 8$ of tetrahedral sites and $1 / 2$ of octahedral sites are occupied by cations A and B, respectively. However, this cubic structure can be distorted when transition metal cations with certain electron configurations (such as $\mathrm{Mn}^{3+}$ with $3 \mathrm{~d}^{4}$ ) occupy the octahedral sites, known as the so-called Jahn-Teller distortion $[14,15]$. For a spinel structure rich in $\mathrm{Mn}^{3+}$, net distortion of these octahedral sites could lead to a unit cell transformation from cubic to tetragonal if the amount of 
$\mathrm{Mn}^{3+}$ in these sites is high enough [16].

To establish whether the dendrites in the glaze surface depict a cubic or tetragonal crystal structure at room temperature, a powder sample was synthesized by heating in a kiln using the same heating program as that used for glaze. The elemental ratio $\mathrm{Mn}$ : Co $: \mathrm{Al}=1: 0.4: 0.15$ was similar to the ratio detected from the dendrites in the glaze (Fig. S2).

The XRD pattern of the powder sample shown in Fig S3 can be indexed to the tetragonal spinel structure with the position of the $(101)_{\mathrm{T}}$ peak matching to the $(111)_{\mathrm{C}}$ peak position in the XRD pattern from the dendrites in the glaze (The subscript $\mathrm{T}$ stands for tetragonal unit cell). This result implies that the dendrites have a tetragonal unit cell with parameters, $\mathrm{a}=5.7790 \AA, \mathrm{c}=8.7358 \AA$. This unit cell was then used to re-index the SAED patterns and HRTEM images recorded from the dendrites. For example, the two adjacent diffraction spots shown in the inset of Fig 3(b), indexed to the $(20 \overline{2})_{C}$ and $(2 \overline{2} 0)_{\mathrm{C}}$ planes of a pseudo-cubic unit cell can now be indexed to the $(11 \overline{2})_{\mathrm{T}}$ and $(200)_{\mathrm{T}}$ planes in the tetragonal spinel with an interplane angle of $58.9^{\circ}$. More HRTEM images (Fig S4) recorded from the dendrites viewed along other directions further confirm that the average structure is tetragonal as determined above. The relation between the pseudo-cubic unit cell and the tetragonal unit cell is shown in Fig. S5. 
With the results obtained in the present work, a possible formation of the imitated Tianmu glaze could be proposed. When the temperature increases, the remaining water in the glaze evaporates. At a certain temperature, potassium feldspar starts to melt and forms a glass body with the principal elements of potassium, silicon, aluminium and oxygen. With a further increase of temperature, the viscosity of the glass decreases continuously and the glass body can be treated as a solvent, dissolving gradually the other solid particles to form a homogeneous liquid mixture.

During a temperature decrease from a high temperature, in many glass systems such as $\mathrm{MgO}-\mathrm{SiO}_{2}, \mathrm{CaO}-\mathrm{SiO}_{2}$ or $\mathrm{BaO}-\mathrm{TiO}_{2}-\mathrm{SiO}_{2}$, a common phenomenon known as liquid phase separation can be observed depending on the mixing ratio $[17,18]$. This phenomenon can be explained by the shape and the size of cation-oxygen polyhedra formed by silicon or other cations $[19,20]$. If the shape and the size of a polyhedron formed by other cations in the glass are similar to the $\mathrm{Si}-\mathrm{O}$ polyhedron, the whole system tends to form a single phase without a phase separation. On the other hand, if the polyhedra formed by other cations are significantly different from the Si-O polyhedron, these cations would tend to be repulsed from the glass and aggregate into other phases. This process obeys the principle: 'like attracts like'. In the present work, the trace for liquid phase separation was observed as the spherical clusters separated from the glass 
matrix as shown in Fig 2(c).

These clusters are rich in manganese, cobalt, vanadium, tungsten and bismuth, none of which are present in the glass matrix, while the concentrations of $\mathrm{Si}$ and $\mathrm{Al}$ in the clusters are very low (Fig. 4). A close examination of the elemental distribution in the cluster reveals that the elemental dispersion in some of the clusters are not even, which might be caused by further phase separation or crystal segregation in the clusters. HRTEM images of the clusters show very small nanocrystallites embedded in the amorphous matrix (Fig. S6a) which are difficult to be detected by XRD. The d-spacing $(9.74 \AA)$ measured from the nanocrystallite in the inset of Fig. S6a is typical for metal oxides rather than metals. Since a cluster includes several elements, the nanocrystallites are not simple metal oxides. It has been well known that many transition metal oxides can form solid solutions with $\mathrm{Bi}_{2} \mathrm{O}_{3}$ crystal [21-23], implying that many nanocrystals in the glaze are bismuth oxide based solid solutions. It is also noted that the initial phase separation resulted in much smaller clusters of transition metal oxides as observed on the surface of the large clusters. The large clusters formed due to further aggregation of these nanocrystallites, which underwent re-crystallization into larger crystals (Fig. 4, S6b).

After the liquid phase separation, a thin surface layer with abundant 
nanocrystallites became the most favorable site for crystallization of $\mathrm{Mn}-$, Co- and Alcontaining spinel during the cooling process, and the air-liquid interface favored heterogeneous nucleation [24]. When the temperature started to decrease, several nuclei with a critical radius were generated in this layer after the energy barrier for nucleation had been overcome. These nuclei then become the original point for hierarchical growth of the spinel dendrites. During the growth process, the dendrites kept consuming the manganese, cobalt and aluminium source in the film and expanded along six equivalent $<2 \overline{2} 0>_{\mathrm{C}}$ directions with the most stable (111) facet parallel to the glaze surface.

Other elements in the film which were not suitable for the spinel crystal structure were pushed away from the dendrites. The K-rich layers on both the top and bottom sides of the dendrite layer are evidence of this elemental re-distribution. These unreacted materials would also accumulate in the boundaries between adjacent dendrites as seen in Fig. 5. Since there is no space for the continuous 2-dimensional growth in the boundaries, the remaining manganese, cobalt and aluminium in the film would start to grow locally, forming the thick edges along the boundaries. The unreacted materials (vanadium, tungsten and bismuth, etc.) would also accumulate in the boundary areas. Elemental mapping recorded from top view and cross section of a boundary shown in Fig. S7 further supports this hypothesis. 
Colour appearance from the 2D spinel dendrite of the glaze is also investigated in the present work. A commercial Tianmu bowl, whose glaze has been confirmed to possess similar components to the synthetic Tianmu glaze, has been used for the comparison study because of its rainbow-like colour appearance. The surface dendrites have a tetragonal spinel structure with a similar composition compared to the dendrites in the synthetic glaze (Fig. S8-S10). The results show that the light interference could be the principal factor for the colour appearance on the glaze surface.

Fig. 6(a-d) show the colour appearance recorded by optical microscopy from the commercial Tianmu bowl. All of these images include at least one triangular shaped dendrite recorded from different locations from the edge (Fig. 6a) to the center of the bowl (Fig. 6d), and the images in Fig. 6(b,c) were recorded from areas in between. Fig 6(e) was recorded from the original synthetic Tianmu glaze, while Fig 6(f) was recorded from a re-fired synthetic sample, for which the temperature was increased at $4{ }^{\circ} \mathrm{C} \min ^{-1}$ to $800{ }^{\circ} \mathrm{C}$, followed by $2{ }^{\circ} \mathrm{C} \min ^{-1}$ to $1000{ }^{\circ} \mathrm{C}$. It is noted that the six-fold dendrites exist in both glazes (Fig. 6d,e). These images show that, although the main crystal structure on the glaze surface is spinel, its colour appearance can be very different.

On the commercial Tianmu bowl, the colour change is more obvious from the edge to the center. The result shows that the main difference between different areas of the 
bowl is the thickness of the crystalline layer as shown in Fig. 7. The thickness of the crystal near the edge of the bowl is about $88 \mathrm{~nm}$ (Fig. 7a) and significantly increases to approximately $179 \mathrm{~nm}$ in the middle region (Fig. 7b), and further to about $235 \mathrm{~nm}$ near the center (Fig. 7c). The line chart of the measured values of dendrite thickness from the edge to the center of the bowl shown in Fig. 7(d) clearly displays this tendency. This trend of thickness change, corresponding to the colour change shown in Fig. 6(a-d), implies that the thickness of crystalline layer plays an important role for the colour appearance, and that the performance is similar to the light interference. Fig. 7(e) is a light spectrum which demonstrates the colour of interference light coordinates to the thickness of a thin film with the thickness increasing from left to right. The continuous colour change from the spectrum is almost identical to the colour change observed from the edge to the center of the commercial Tianmu bowl. Based on these results, the relationship between the thickness of the crystal and the colour appearance can be roughly determined, and the colour range shown in Fig. 6(a-d) can be marked as the four blue lines shown on the spectrum in Fig. 7(e). After analysis of the commercial Tianmu bowl, the thickness of the dendrites in the synthesized glaze on a flat substrate was also measured from profile SEM images. The mean value of the dendrite thickness in the as-synthesized glaze is about $88 \mathrm{~nm}$ and that in the re-fired glaze is about $290 \mathrm{~nm}$. 
The rough colour ranges for both samples based on the thickness were marked by the two black lines on the spectrum in Fig. 7(e), which also matches to the colour appearance in Fig. 6(e) and 6(f).

The main reason that causes the increasing thickness of the crystal layer from the edge to the center of the commercial Tianmu bowl is gravity. Since the whole glaze layer became soft and flowable during firing at high temperature, the $\mathrm{Mn}$ - and Cocontaining clusters in the glaze would flow over the surface, forming a surface layer with different thicknesses depending on the curvature of the bowl, leading to a colour change. When the Tianmu bowl is rotated, i.e. the view direction to the glaze surface changes, the colourful patterns moves. This is another evidence to support the light interference mechanism. On the other hand, the synthesized glaze on a flat substrate has a relatively uniform dendrite thickness. The colour change is not so significant. These results show that, with a proper control of synthetic method, one might be able to control the distribution of the crystal thickness on glaze and therefore the colour appearance on the Tianmu bowls. The more complex local colour patterns depend on the local microstructures.

\section{Conclusion}

According to the investigation of glaze samples of a commercial Tianmu bowl and 
that synthesized on a flat substrate, pseudo-cubic spinel dendrite crystals form on the glaze surface with highly selective growth directions of $\left\langle 2 \overline{2} 0>_{C}\right.$. The distortion of spinel structure is due to the solid solution nature with an average ratio of $\mathrm{Mn}: \mathrm{Co}: \mathrm{Al}=1$ : $0.4: 0.15$. The colour appearance of the glaze is mainly governed by light interference on the dendrite layer with different thicknesses, which is significantly changed by many factors such as the viscosity of the glaze, the program for firing and the curvature of the bowls. Liquid phase separation in the glaze at high temperature lead to spherical clusters of mixed oxides of $\mathrm{Bi}$ and several added transition metals. The clusters are embedded deeply in the glaze which contribute to the black or dark brown background colour. This work sheds light on understanding the crystal formation in Tianmu glazes, and would be beneficial to future design of glazes with wonderful colour performances.

\section{Acknowledgements}

We gratefully acknowledge Dr Tomohiro Mihira and Dr Misumi Kadoi from the Ion Beam Application Group and Mr Akira Yasuhara from EM Application Department at JEOL Ltd. for TEM specimen preparation and elemental mapping data collection, Dr. Emrah Yücelen at FEI Ltd. for the collection of TEM images and EDX elemental mappings from the clusters. CYC would like to thank Mr. Yi-Zhi Qiu at Shang Liu Pottery Club for the recipe of Tianmu glaze, Dr. David Miller for his help to prepare the 
ion-milled TEM samples, Mr. Ross Blackley for the help on using SEM and TEM microscope. WZZ thanks EPSRC for financial support on FEG-SEM equipment (EP/F019580/1). 
References

[1] S.G. Valenstein, A handbook of Chinese ceramics, Metropolitan Museum of Art, New York (2012).

[2] H. Nishida, S. Sato, Tenmoku, Heibonsha, Tokyo (Japanese Edi) (1999).

[3] X. Cai, The record of tea (Chinese Edi) (about 1049).

[4] Collection in Seikado Bunko Art Museum

(http://www.seikado.or.jp/e 040000.html).

[5] Collection in The Museum of Oriental Ceramics, OSAHA

(http://www.moco.or.jp/en/index.php).

[6] Y. Kanq, Analyzing the development and their decorative features of tea-calices with black glaze in JiZhou Kiln, J. Nanjing Arts Inst. Fine Art \& Design (Chinese Edi) 2 (2005) 88-89.

[7] W. Li, H. Luo, J. Li, J. Li, J. Guo, Studies on the microstructure of the black-glazed bowl sherds excavated from the Jian Kiln site of ancient china, Ceram. Int. 34 (2008) 1473-1480.

[8] Y. Dai, Application and study of kiln transformation black glaze, China Ceramics (Chinese Edi) 7 (2006) 80-83.

[9] H. Ye, Yao-bian Temmoku black glaze china, Bull. Chinese Ceramic Soc. (Chinese Edi) 3 (1982) 15-48. 
[10] J. Wang, W. Liu, B. Liu, Z. Li, Study on simulation of oil spot glaze in the Song Dynasty, Ceramics Engineering (Chinese Edi) 6 (2001) 13-16.

[11] R. Huang, X. Chen, S. Chen, D. Zhao, J. Wang, R. Lu, N. Zhang, A high resolution electron microscopic investigation on the imitative glaze of Yaobian Temmoku in Southern Song Dynasty, China Ceramics (Chinese Edi) 1 (1988) 17-21.

[12] Z. Yu, Crystallization glaze, Foshan Ceramics (Chinese Edi) 7 (2003) 21-23.

[13] G.M. Faulring, W.K. Zwicker, W.D. Forgeng, Thermal transformations and properties of cryptomelane, Am. Mineral. 45 (1960) 946-959.

[14] H.A. Jahn, E. Teller, Stability of polyatomic molecules in degenerate electronic states. I. Orbital degeneracy, Proc. R. Soc. London, Ser. A 161 (1937) 220-235.

[15] H. Bordeneuve, C. Tenailleau, S. Guillemet-Fritsch, R. Smith, E. Suard, A. Rousset, Structural variations and cation distributions in $\mathrm{Mn}_{3-\mathrm{x}} \mathrm{Co}_{\mathrm{x}} \mathrm{O}_{4}(0 \leq \mathrm{x} \leq 3)$ dense ceramics using neutron diffraction data, Solid State Sci. 12 (2010) 379-386.

[16] S. Naka, M. Inagaki, T. Tanaka, On the formation of solid solution in $\mathrm{Co}_{3-\mathrm{x}} \mathrm{Mn}_{\mathrm{x}} \mathrm{O}_{4}$ system, J. Mater. Sci. 7 (1972) 441-444.

[17] P.F. James, Liquid-phase separation in glass forming systems, J. Mater. Sci. 10 (1975) 1802-1825.

[18] W. Li, J. Li, J. Wu, J. Guo, Study on the phase-separated opaque glaze in ancient 
China from Qionglai kiln, Ceram. Int. 29 (2003) 933-937.

[19] E.M. Levin, S. Block, Structural interpretation of immiscibility in oxide systems: I, Analysis and calculation of immiscibility, J. Am. Ceram. Soc. 40 (1957) 95-106.

[20] S. Block, E.M. Levin, Structural interpretation of immiscibility in oxide systems:

II, Coordination principles applied to immiscibility, J. Am. Ceram. Soc. 40 (1957) $113-118$.

[21] W.Z. Zhou, Defect fluorite-related superstructures in the $\mathrm{Bi}_{2} \mathrm{O}_{3}-\mathrm{V}_{2} \mathrm{O}_{5}$ system. J. Solid State Chem. 76 (1988) 290-300.

[22] W.Z. Zhou, Defect fluorite superstructures in the $\mathrm{Bi}_{2} \mathrm{O}_{3}-\mathrm{WO}_{3}$ system. J. Solid State Chem. 108 (1994) 381-394.

[23] W.Z. Zhou, Microstructures of some Bi-W-Nb-O phases. J. Solid State Chem. 163 (2002) 479-483.

[24] R.P. Sear, Nucleation: Theory and applications to protein solutions and colloidal suspensions, J. Phys. Condens. Matter 19 (2007) 033101. 
Figure captions:

Fig. 1. Mimetic Tianmu bowls made by Shang Liu Pottery Club in Taiwan, (a) Yao Bian Tianmu with many 'eyes', (b) Oil Spot Tianmu, and (c) Yao Bian Tianmu with small number of 'eyes'.

Fig. 2. (a) SEM image recorded from the top view of the glaze. (b) Enlarged SEM image of part of (a). (c) Dark field TEM profile image of the glaze near the surface, followed by elemental mappings. The arrow marked in the image points out the surface of the glaze.

Fig. 3. (a) XRD patterns of the as prepared glaze and the glaze specimen with the dendrite layer removed. (b) A single crystalline dendrite and its corresponding SAED pattern recorded from the $[111]_{\mathrm{C}}$ view direction of the pseudo-cubic spinel structure. The diffraction spots in a hexagonal pattern marked by yellow circles are indexed to $\{2 \overline{2} 0\}_{\text {c planes. }}$

Fig. 4. HAADF image followed by elemental maps recorded from a spherical particle in the glaze with a diameter of about $200 \mathrm{~nm}$.

Fig. 5. SEM images of a boundary marked between two white dotted lines between adjacent dendrites viewed down (a) the top surface and (b) the cross section. The boundary is composed by thick edges of the dendrites and irregular shaped structures 
rich in the unreacted materials.

Fig. 6. (a-d) Optical microscopic images recorded from a commercial Tianmu bowl at different locations from the bowl edge to the bowl center. $(\mathrm{e}, \mathrm{f})$ Optical microscopic images recorded from the freshly synthesized glaze without and with the re-firing process.

Fig. 7. Backscattered SEM images showing crystalline layer (marked between two arrows) on the surface tends to increase in thickness from (a) the edge to (c) the center of the bowl. (d) Line chart showing the measured thickness (unit: $\mathrm{nm}$ ) from the bowl. (e) Light interference spectrum coordinated to a thin film with the thickness increasing from left to right. The lines marked on the spectrum are the ranges of colour appearance relative to Fig. 6(a-f). 
Table 1: Components and purity of the raw materials and their expected functions in the glaze formation.

\begin{tabular}{|c|c|c|c|}
\hline Material & Purity $(w t \%)$ & $\begin{array}{l}\text { Amount } \\
\text { (g) }\end{array}$ & Function \\
\hline $\begin{array}{l}\text { potassium } \\
\text { feldspar }\end{array}$ & $\begin{array}{l}\mathrm{SiO}_{2} \text { 66.28, } \mathrm{Al}_{2} \mathrm{O}_{3} \quad 18.87, \mathrm{CaO} \\
0.131, \\
\mathrm{~K}_{2} \mathrm{O} \text { 11.18, } \mathrm{Na}_{2} \mathrm{O} \text { 2.884, Others < } \\
0.1\end{array}$ & 35.625 & Main glaze body \\
\hline $\mathrm{SiO}_{2}$ & $\mathrm{SiO}_{2}$ 99.4, $\mathrm{Al}_{2} \mathrm{O}_{3} 0.35$, Others $<0.1$ & 9.375 & Main glaze body \\
\hline $\mathrm{MnO}_{2}$ & $\begin{array}{l}\mathrm{MnO}_{2} 75, \mathrm{Fe}_{2} \mathrm{O}_{3} 5.8, \mathrm{SiO}_{2} 4, \mathrm{Al}_{2} \mathrm{O}_{3} \\
\text { 2.9, } \mathrm{BaO} 1.4, \mathrm{~K}_{2} \mathrm{O} \text { 0.8, Others }<0.1\end{array}$ & 18.75 & Colorant \\
\hline $\mathrm{CoO}$ & $\mathrm{CoO} 47.5, \mathrm{Co}_{3} \mathrm{O}_{4} 47.5$, Others $<5$ & 3.125 & Colorant \\
\hline $\mathrm{Bi}_{2} \mathrm{O}_{3}$ & 99.9995 & 18.75 & Glaze enhancer \\
\hline $\mathrm{NH}_{4} \mathrm{VO}_{3}$ & 98.6 & 3.75 & Flux \\
\hline $\mathrm{WO}_{3}$ & 99.998 & 9.375 & Increase viscosity \\
\hline $\mathrm{MgCO}_{3}$ & $>95$, moisture $<3$ & 1.25 & Flux \\
\hline ISOBAM & & 0.5 & Binder \\
\hline
\end{tabular}
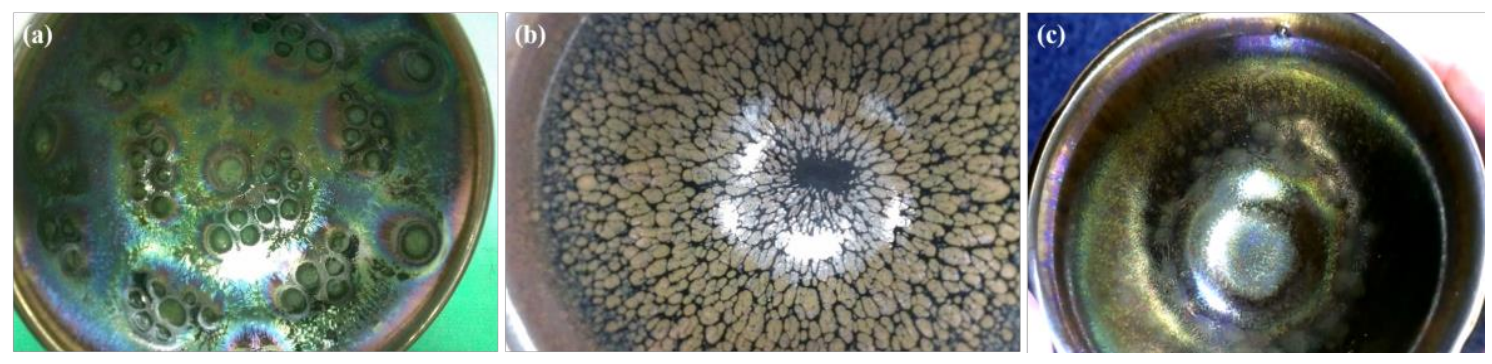

Figure 1 

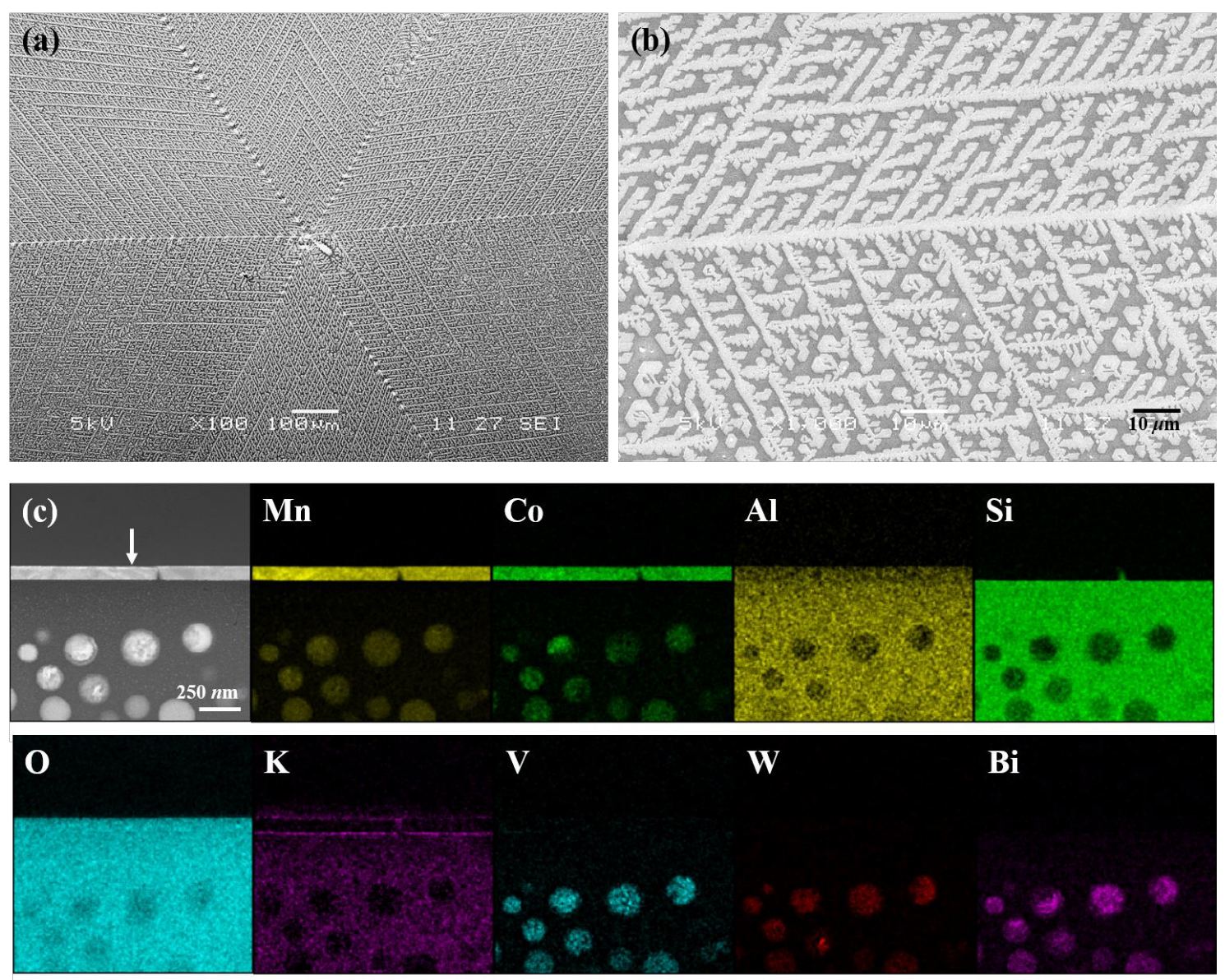

Figure 2

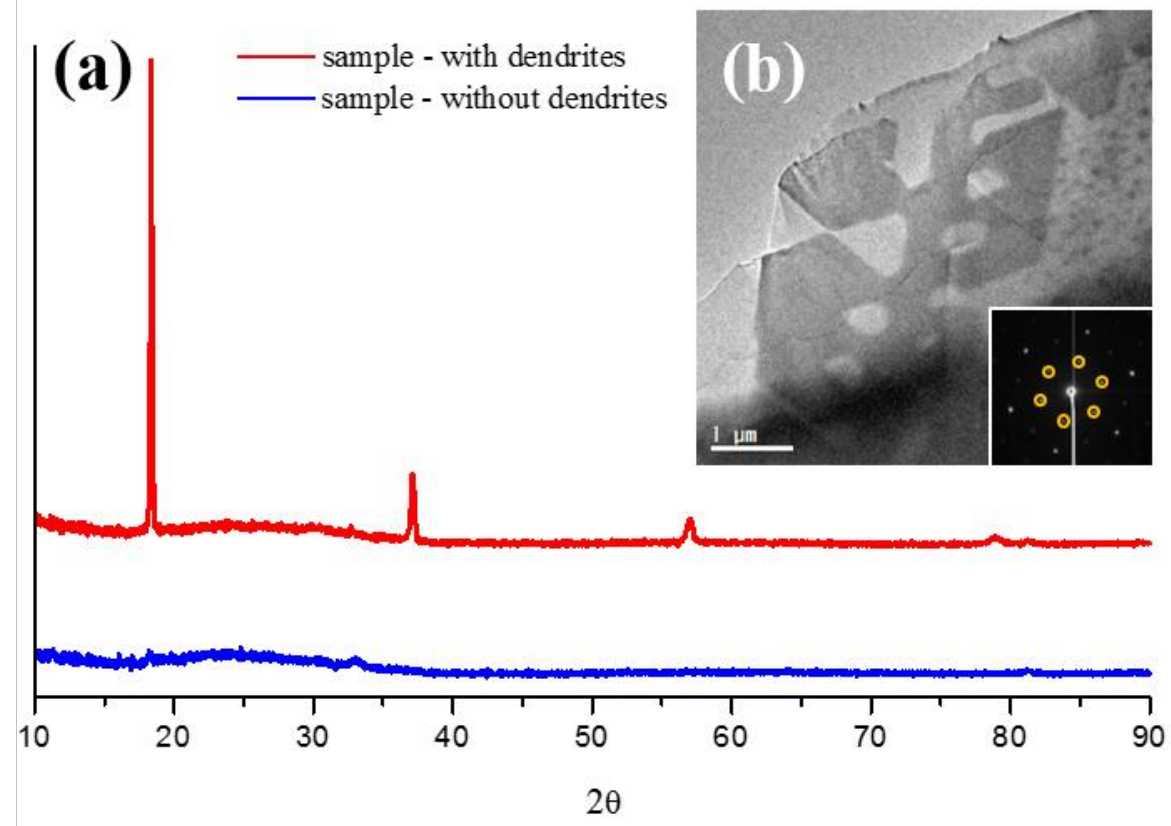

Figure 3 


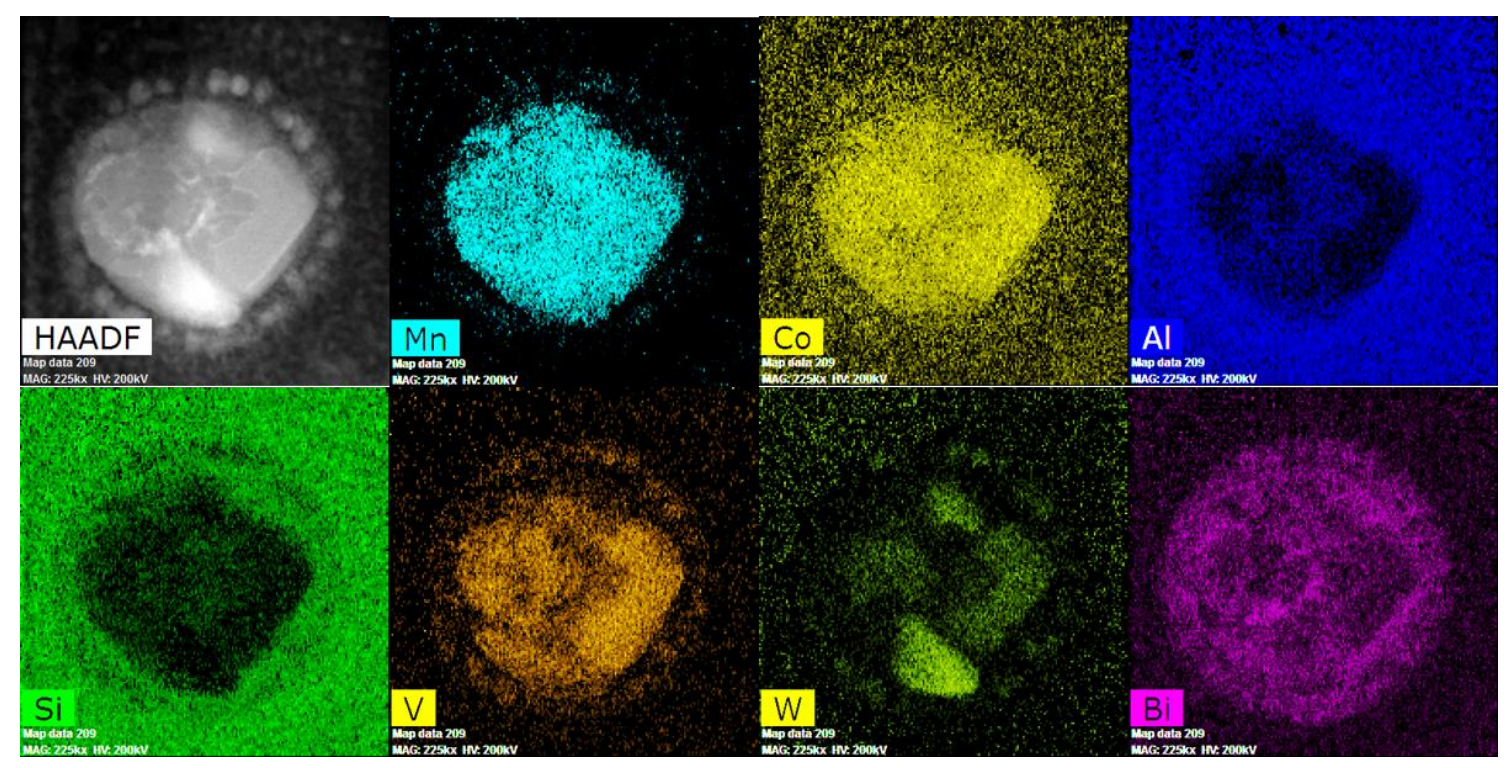

Figure 4

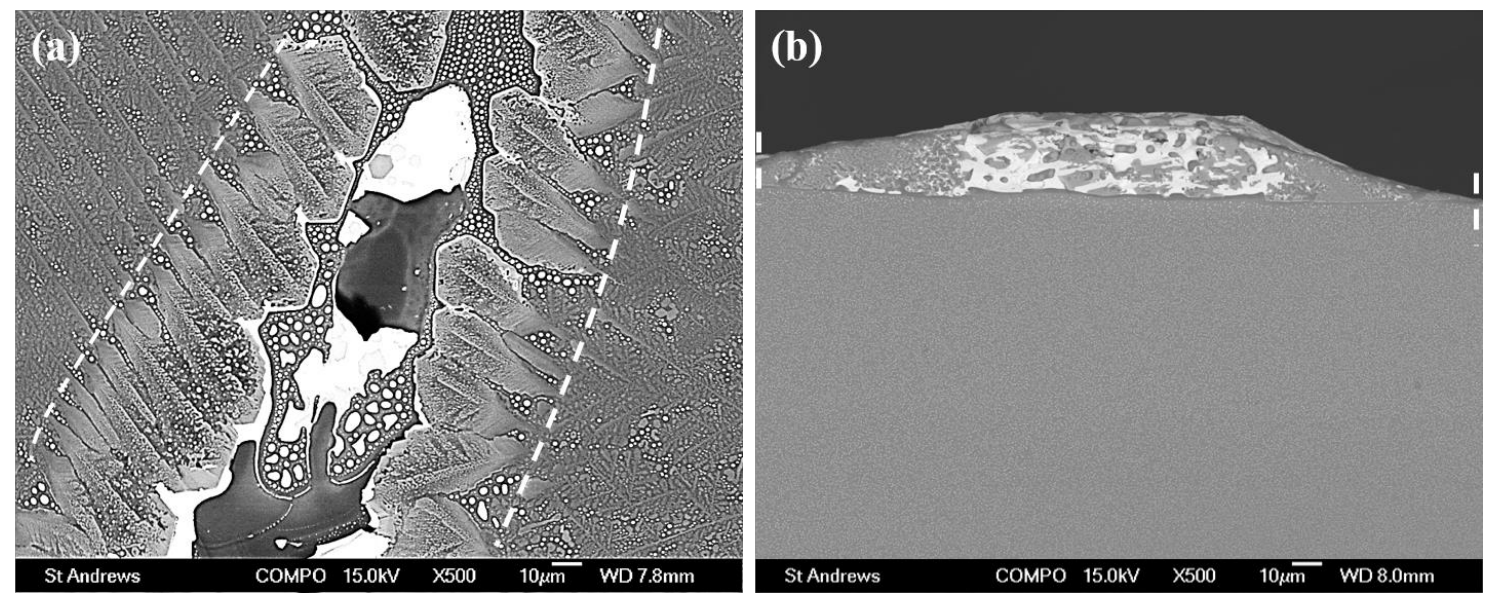

Figure 5 


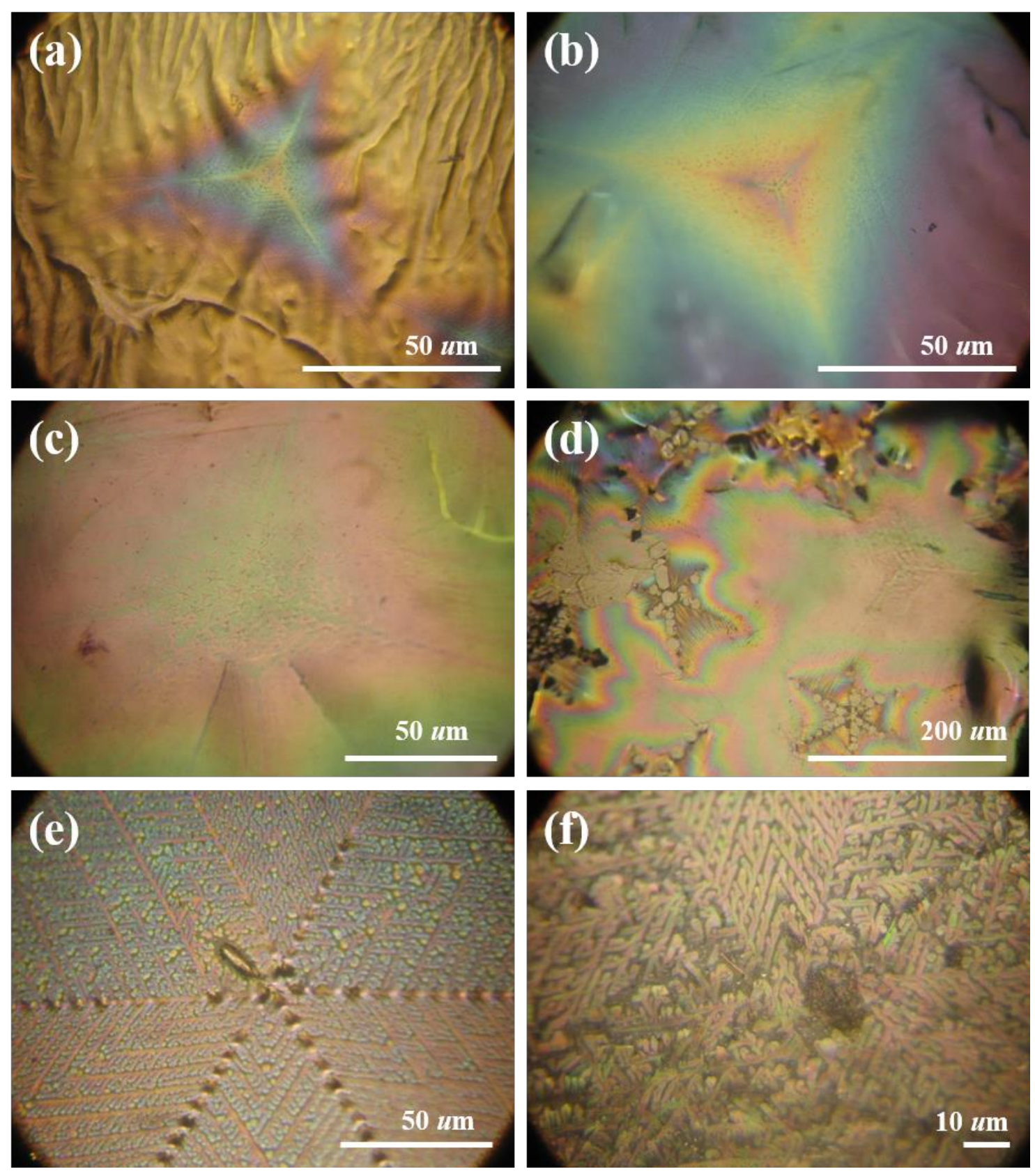

Figure 6 


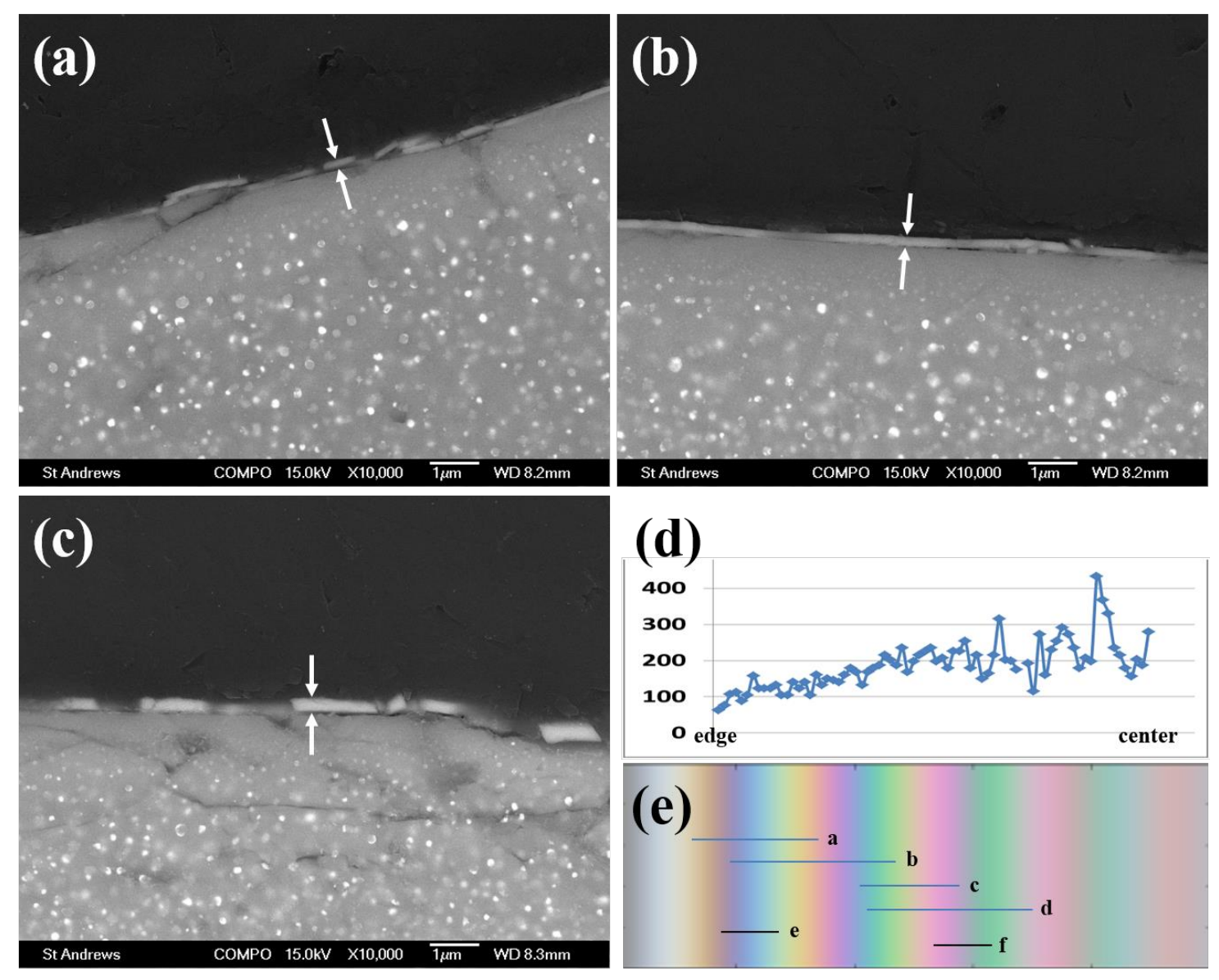

Figure 7 